TERRA. Revista de Desarrollo Local e-ISSN: 2386-9968

Número 9 (2021), 160-174

DOI 10.7203/terra.9.21971

IIDL - Instituto Interuniversitario de Desarrollo Local

\title{
Participando desde la infancia en la toma de decisiones públicas. Experiencia del municipio de Rafelbunyol (Valencia, España)
}

\author{
Agar Moreno Secaduras \\ Coordinadora de Gobierno Abierto del Ayto. de Rafelbunyol (Valencia, España) \\ a.m.secaduras@gmail.com
}

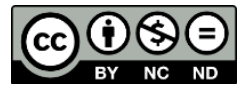

Esta obra se distribuye con la licencia Creative Commons Reconocimiento-NoComercial-SinObraDerivada 4.0 Internacional 


\section{SECCIÓN EXPERIENCIAS Y BUENAS PRÁCTICAS}

\section{Participando desde la infancia en la toma de decisiones públicas. Experiencia del municipio de Rafelbunyol (Valencia, España)}

Resumen: La participación ciudadana constituye uno de los tres pilares del Gobierno Abierto. La transformación en la manera de gobernar y gestionar las administraciones públicas bajo estos preceptos e incorporando, por tanto, la participación ciudadana de una manera transversal, implica un cambio cultural no solo en las instituciones sino también en la ciudadanía. Para ello deviene fundamental incidir en las generaciones más jóvenes de nuestra sociedad, pero esto contrasta con una tradicional exclusión por razón de edad del colectivo infantil en los procesos participativos de gestión y toma de decisiones públicas, debido a una consideración de la infancia como inexperta para decidir sobre sobre los aspectos que les afectan y sobre los de sus comunidades. En contraposición, este artículo se adentra en la experiencia de participación ciudadana infantil 'Participando desde la infancia', llevada a cabo por el Ayuntamiento de Rafelbunyol (Valencia), con la finalidad de fomentar, desde una perspectiva pedagógica, la práctica de hábitos democráticos relativos a la participación entre el colectivo infantil, favorecer la participación ciudadana en la gestión pública a edades tempranas, mitigar esa tradicional exclusión por razón de edad de la población infantil en la toma de decisiones públicas y, por tanto, fortalecer el empoderamiento ciudadano y generar una cultura de gobernanza participativa desde la infancia.

Palabras clave: Gobierno Abierto, infancia, participación ciudadana infantil, gobernanza participativa, empoderamiento ciudadano.

Recibido: 22 de noviembre de 2021

Devuelto para revisión: -

Aceptado: 23 de noviembre de 2021

\section{Referencia / Citation:}

Moreno, A. (2021). Participando desde la infancia en la toma de decisiones públicas. Experiencia del municipio de Rafelbunyol (Valencia, España). TERRA. Revista de Desarrollo Local, (9), 160-174. DOI 10.7203/terra.9.21971 


\section{INTRODUCCIÓN}

El Gobierno Abierto es un modelo o paradigma sobre cómo gobernar y gestionar las Administraciones Públicas basado en tres principios fundamentales:

- La participación de la ciudadanía en la toma de decisiones públicas y en la formulación de políticas.

- La transparencia pública.

- La colaboración de las Administraciones entre ellas y con diferentes sectores de la sociedad.

Centrando la atención en la participación ciudadana, cabe apuntar que el ámbito local es el nivel de gobierno más próximo a la ciudadanía, lo que propicia que este principio del Gobierno Abierto encuentre en el entorno local mayores afinidades para su desarrollo e implementación.

No obstante, la transformación en la manera de gobernar y gestionar las administraciones públicas, incorporando la participación de la ciudadanía de una manera transversal, es un proceso complejo que implica un cambio cultural no solo en las instituciones, sino también en la ciudadanía, y en esto último, deviene fundamental incidir tanto en la formación como en la información de las personas más jóvenes de nuestra sociedad, que serán quienes practicarán estas nuevas formas de gobierno (Mayor et al., 2010).

Sin embargo, durante los últimos treinta años, las dinámicas participativas en España han marginado a los más jóvenes -lo cual, además, contrasta con el Artículo 9 de la Constitución Española, que establece que los poderes públicos facilitarán " $l a$ participación de todos los ciudadanos en la vida política, económica, cultural y social" (Mayor et al., 2010, p.12), debido a la tradicional representación social que se tiene de la infancia como un colectivo inexperto que aún no se encuentra capacitado para decidir sobre los aspectos que le afectan ni sobre sus comunidades, considerándolos, por tanto, como pre-ciudadanos.

En contraposición, a finales de los años noventa, surge todo un movimiento a favor de que la infancia tenga un rol principal en la participación ciudadana, conocido como 'Participación Protagónica', y entendido como el proceso social por el cual los niños y niñas han de ser los protagonistas de su desarrollo y el de su comunidad, garantizando plenamente sus derechos (Novella, 2012).

En este sentido, cabe apuntar que la Convención sobre los Derechos del Niño (UNICEF, 2015, p.23) establece en su Artículo 12.1 la participación infantil como uno de los derechos de este colectivo: "los Estados Partes garantizarán al niño que esté en condiciones de formarse un juicio propio el derecho de expresar su opinión libremente en todos los asuntos que afectan al niño, teniéndose debidamente en cuenta las opiniones del niño, en función de la edad y madurez del niño".

Para conseguir que la infancia tenga un rol principal en la participación ciudadana y garantizar, por tanto, su derecho a participar, es necesario:

- Incorporar al colectivo infantil en la concepción social de ciudadanía activa, entendida, según Puig (2005) como aquella que contribuye al bien común, cumple con sus deberes y exige sus derechos.

- Desarrollar oportunidades que permitan incluir a los niños y niñas en las prácticas democráticas. 
- Diseñar proyectos y actuaciones, desde una perspectiva pedagógica, mediante las cuales la infancia intervenga en los procesos participativos de toma de decisiones públicas y de desarrollo comunitario, especialmente en aquellos asuntos que les afectan, aprendiendo así a participar participando.

La infancia es una etapa fundamental en la socialización y en el aprendizaje social, por lo que la práctica de hábitos democráticos a edades tempranas, y la misma participación de niños y niñas en la gestión de lo público y en la toma de decisiones, constituyen una oportunidad para su desarrollo personal, pero también, social, comunitario y, por tanto, ciudadano.

En este sentido, apuntar que la participación infantil, entendida también como práctica pedagógica, permitirá fomentar entre los niños y niñas su autonomía ciudadana, de una manera cada vez más responsable y comprometida a medida que se impliquen en experiencias participativas (Novela, 2012), favoreciéndose así la construcción de valores democráticos y de una ciudadanía empoderada desde edades tempranas.

\section{2. ÁREA DE ACTUACIÓN Y CONTEXTUALIZACIÓN DE LA EXPERIENCIA}

La experiencia presentada se implementó en el municipio de Rafelbunyol, perteneciente al área metropolitana de la ciudad de Valencia (España) y situado en la Comarca de l'Horta Nord, y que en el año 2019 contaba con 9.123 habitantes ${ }^{1}$, de los cuales, el 20,6\% (1.879 personas) eran menores de 18 años.

En la legislatura de 2015, el Ayuntamiento de Rafelbunyol inició un proceso de cambio cultural institucional basado en los principios del Gobierno Abierto, con la creación de las primeras Concejalías de Participación Ciudadana y de Transparencia y la elaboración de normas municipales, así como la realización de diferentes actuaciones y procesos, que promovían la transparencia de la gestión pública y la democracia participativa.

Asimismo, en la legislatura de 2019, las Concejalías de Participación Ciudadana y de Transparencia se aúnan bajo la Concejalía de Gobierno Abierto y se crea también la primera Concejalía de Infancia y Adolescencia, con la finalidad de garantizar los derechos de este colectivo y de mejorar el protagonismo de los niños, niñas y adolescentes en las políticas, programas y decisiones públicas, no solo como destinatarios de las mismas, sino también como cocreadores de ellas.

\section{JUSTIFICACIÓN Y OBJETIVOS DE LA EXPERIENCIA}

En octubre de 2019 se lleva a cabo desde las Concejalías de Gobierno Abierto y de Infancia y Adolescencia del Ayuntamiento de Rafelbunyol un análisis de la realidad participativa de la población infantil y adolescente del municipio, detectándose, principalmente:

- Una ausencia generalizada de la participación de la infancia y adolescencia en la toma de decisiones públicas.

\footnotetext{
${ }^{1}$ Datos referentes a 1 de enero de 2019 (Fuente: Instituto Nacional de Estadística, INE).
} 
- Una ausencia generalizada de actuaciones participativas dirigidas a la población infantil.

- La inexistencia de espacios formales de participación ciudadana directamente relacionados con la infancia y adolescencia del municipio.

Estos resultados evidenciaron la necesidad de trazar una estrategia dirigida a la infancia y adolescencia del municipio que promoviese la participación de este colectivo -que en el año 2019 representaba el 20,6\% de la población total (Padrón) - en los procesos de toma de decisiones públicas y en la gestión municipal y con los siguientes objetivos:

- Garantizar la participación ciudadana de la infancia y la adolescencia en la gestión pública.

- Mitigar la tradicional exclusión por razón de edad de la población infantil en la toma de decisiones públicas.

- Inculcar la práctica de hábitos democráticos relativos a la participación entre el colectivo infantil, desde una perspectiva pedagógica.

- Fomentar la implicación ciudadana activa en las decisiones públicas desde edades tempranas.

- Fortalecer el empoderamiento ciudadano y generar una cultura de gobernanza participativa desde la infancia.

\section{DESARROLLO DE LA EXPERIENCIA Y RESULTADOS}

La estrategia 'Participando desde la infancia en la toma de decisiones públicas', llevada a cabo por el Ayuntamiento de Rafelbunyol entre finales del año 2019 y el año 2020, contempló la realización de una serie de actuaciones en torno a la participación ciudadana de este colectivo en la gestión municipal, especialmente sobre aquellos aspectos que les afectan y adaptada a las características de la población infantil.

Estas actuaciones son:

a. Elección por parte del alumnado del nombre oficial de su colegio ${ }^{2}$.

b. Constitución del Consejo Local de Infancia y Adolescencia (CLIA) de Rafelbunyol.

c. Dinámicas de grupo infantil en espacios formales de participación.

d. Foro de Participación Infantil y Juvenil.

e. Elección infantil del graffiti de la Guardería Municipal.

f. Presentación pública infantil sobre herramientas de participación.

\subsection{Elección por parte del alumnado del nombre oficial de su colegio}

Entre diciembre de 2019 y enero de 2020 se llevó a cabo un proceso participativo para la determinación del nombre del Colegio Público de Educación Infantil y Primaria n. ${ }^{\circ} 2$ de

\footnotetext{
${ }^{2}$ Actuación finalista de la 14. ${ }^{\text {a }}$ Distinción Internacional en Participación Ciudadana del Observatorio Internacional de Democracia Participativa, año 2020.
} 
Rafelbunyol -que abrió sus puertas en septiembre de 2019 sin una denominación oficialy que si bien incluyó a toda la comunidad educativa con el propósito de implicar a todos los actores relacionados con el centro en cuestión, la decisión última se delegó en los y las estudiantes, con la finalidad de alcanzar los objetivos de la estrategia de participación ciudadana infantil en la que se enmarca esta actuación.

Figura 1. Proceso participativo en el centro

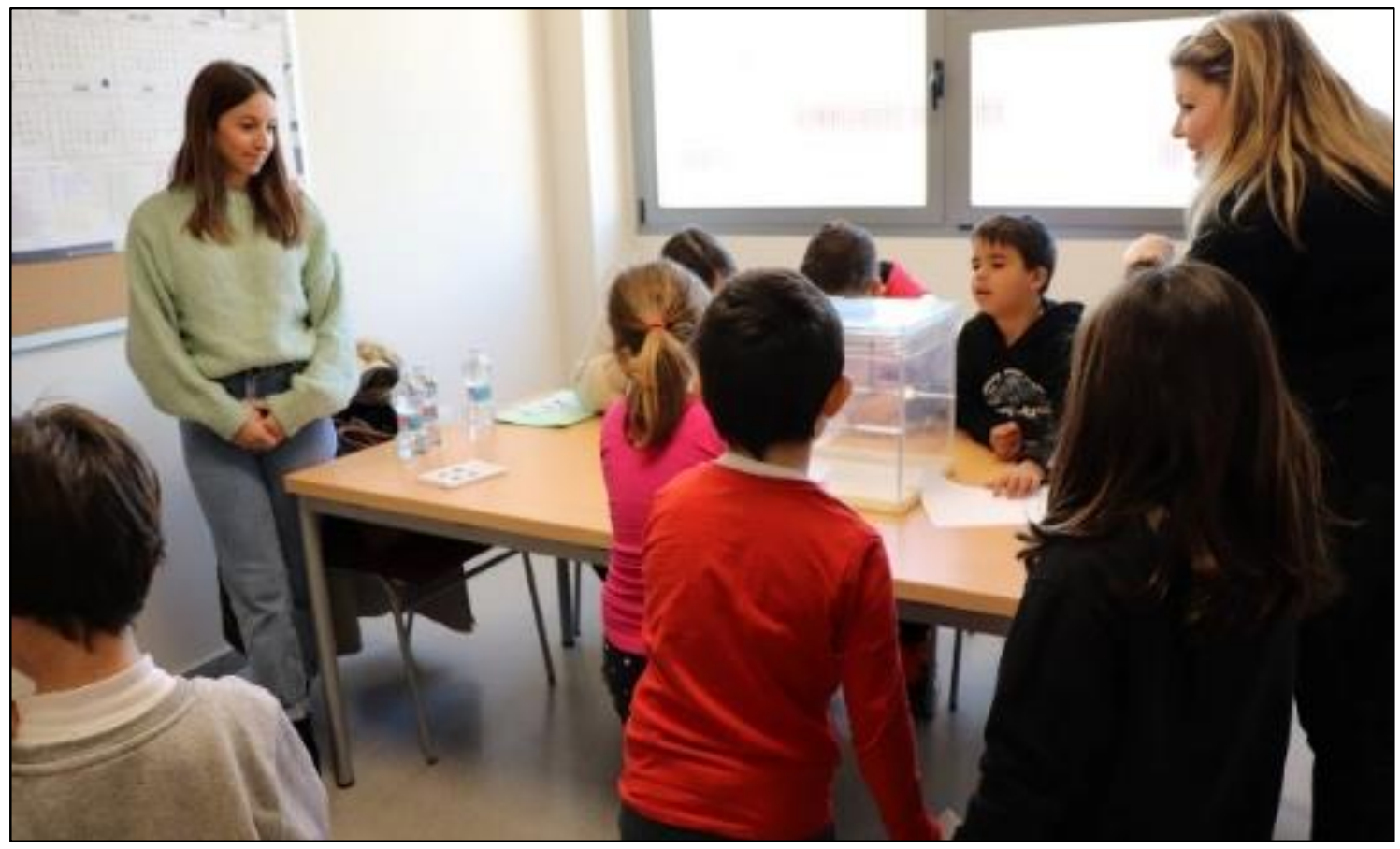

Fuente: elaboración propia.

La denominación de los centros docentes es de gran importancia por los valores que representa. Habitualmente, ésta es una decisión que se toma por los Consejos Escolares o directamente por las propias Administraciones Públicas, por lo que uno de los aspectos más destacables de esta actuación radica en la pionera cesión de esta potestad al alumnado, así como en el impacto sociocultural entre el colectivo infantil y adolescente que ello supone en relación al fortalecimiento del empoderamiento ciudadano desde edades tempranas y a la generación de una cultura de gobernanza participativa entre la infancia.

Para asegurar la efectividad de este proceso participativo, se utilizaron y adaptaron diferentes herramientas o mecanismos de participación, teniendo en cuenta su complejidad en relación a la edad de las personas participantes, lo que dio lugar a que se estructurara en tres fases:

- Una primera fase de propuesta de denominación para el colegio que, dada su mayor complejidad, se delegó en el Consejo Escolar del Centro ${ }^{3}$, conformado por personas adultas, y sobre las que posteriormente votaría el alumnado para la elección definitiva del nombre del colegio.

\footnotetext{
${ }^{3}$ El Consejo Escolar del Centro está compuesto por la dirección y profesorado de éste, las familias del
} alumnado y el Ayuntamiento. 
- Una fase de información previa a la votación infantil y dirigida tanto al alumnado del centro como a sus familias, mediante la cual se les dio a conocer el proceso participativo para la elección de la denominación específica del centro.

Además, para el caso del alumnado, se les repartió unos folletos informativos, diseñados con una apariencia y lenguaje adaptado al colectivo infantil, que contenían cada uno de los nombres de las propuestas de denominación del colegio, una imagen identificativa de cada uno de ellos y una pequeña descripción y el motivo de su relevancia.

- Una tercera fase de votación infantil, mediante la que 372 alumnos y alumnas del centro (el 92,3\% del alumnado total), de entre 3 y 12 años, decidieron el nombre de su colegio.

Figura 2. Propuestas de denominación para el CEIP n² de Rafelbunyol

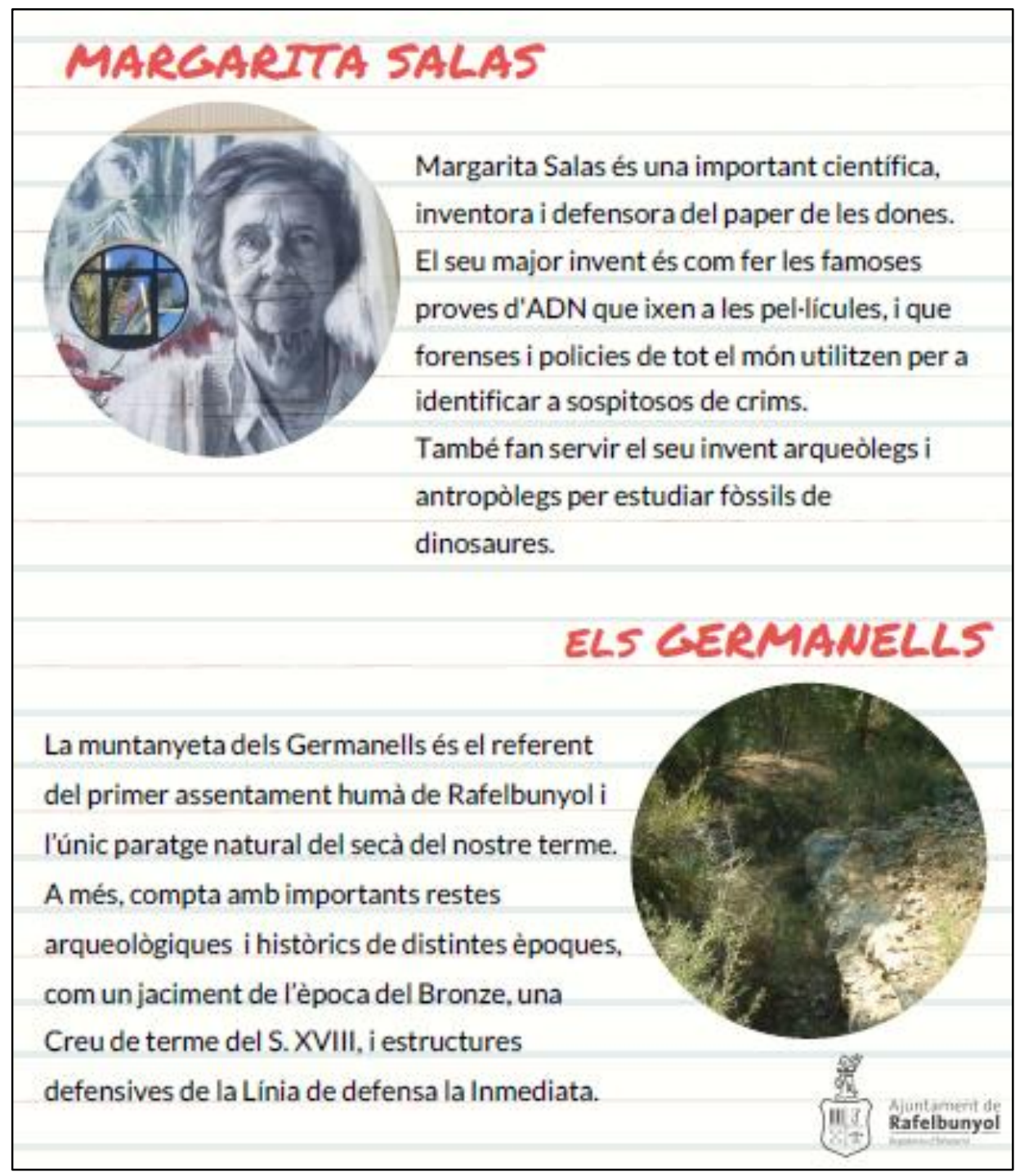

Fuente: elaboración propia. 
Aquí destaca el hecho de que para garantizar la eficacia de la votación y conseguir niveles de igualdad más amplios a la hora de participar, se adaptó el acto de votación a la edad del estudiantado.

Así pues, el alumnado de Educación Primaria, de entre 7 y 12 años, votó seleccionando en una papeleta -también diseñada con una apariencia adaptada y comprensible para el colectivo infantil- una de las cuatro propuestas de denominación, mientras que el alumnado de Educación Infantil, de entre 3 y 6 años, votó el nombre colocándose bajo el cartel de la imagen identificativa de la propuesta, después de que el profesorado les hubiera explicado cada una de las propuestas de denominación.

Además, con el objetivo de continuar promoviendo la práctica de hábitos democráticos entre el colectivo infantil, la jornada de votación estuvo coordinada y asistida también por el alumnado del centro perteneciente al Consejo Local de Infancia y Adolescencia de Rafelbunyol, que se encargó de organizar las votaciones por cursos, explicar a sus compañeros y compañeras como votar, resolver posibles dudas, llevar el control del censo y también participar en el recuento de votos, junto con el resto de personas coordinadoras de la jornada.

Figura 3. Ejemplos del proceso participativo en el ámbito escolar
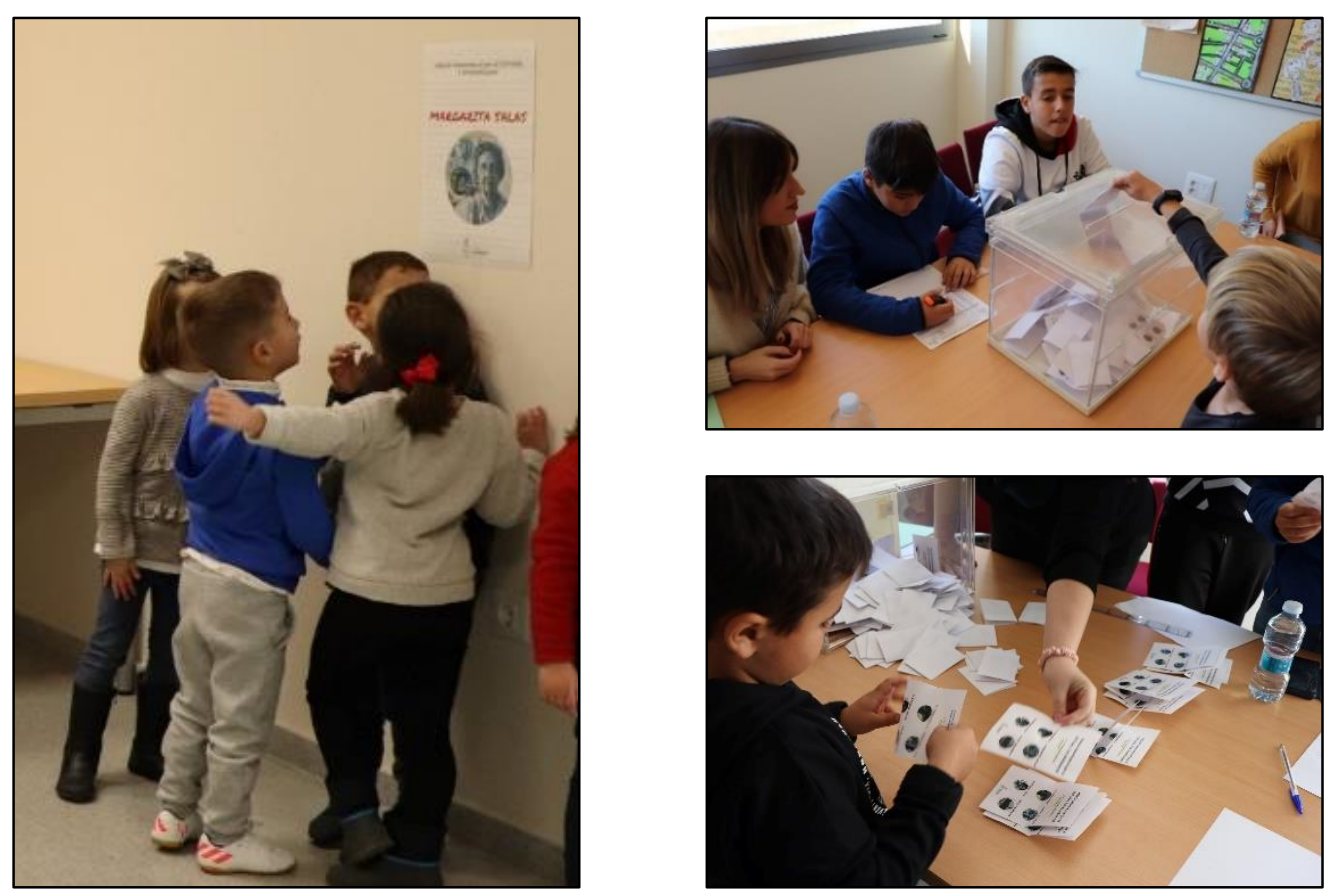

Fuente: elaboración propia.

\subsection{Constitución del Consejo Local de Infancia y Adolescencia (CLIA) de Rafelbunyol}

Uno de los elementos fundamentales para alcanzar los objetivos de la estrategia 'Participando desde la infancia en la toma de decisiones públicas' fue el establecimiento de un órgano de participación municipal en el que estuviera directamente representada la infancia y adolescencia del municipio. 
Figura 4. Acto constitución Consejo Local de Infancia y Adolescencia (CLIA)

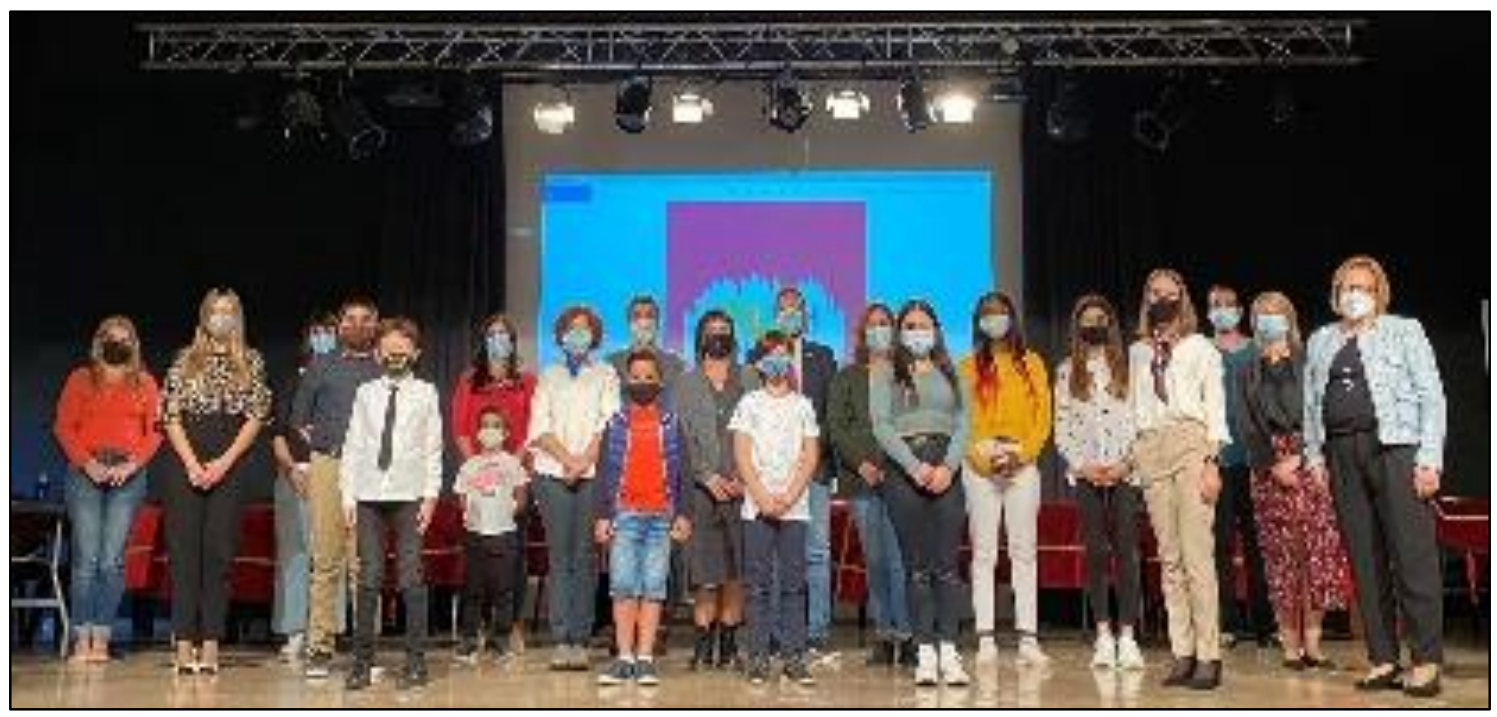

Fuente: elaboración propia.

Entre los años 2019 y 2020 se creó y reguló el primer Consejo Local de Infancia y Adolescencia (CLIA) de Rafelbunyol, conformado por 11 niños y niñas -representantes del colectivo infantil y elegidos por la propia infancia del municipio a través de un proceso de votación realizado en los tres centros educativos de la localidad-, por el Alcalde, la Concejala de Infancia y Adolescencia, representantes de los partidos políticos de la corporación municipal, personal técnico municipal y representantes de las AMPA's de la localidad.

\subsection{Dinámicas de grupo infantil en espacios formales de participación}

Asimismo, esta estrategia también contempló la creación de un Grupo de Trabajo Infantil del CLIA, conformado por los niños y niñas que integran este órgano de participación municipal, como un espacio estable y formal de debate, intercambio de ideas y participación en la toma de decisiones y en diferentes aspectos de la gestión municipal, que se reúne, aproximadamente, una vez al mes en período lectivo.

Con la finalidad de no paralizar el funcionamiento de este Grupo de Trabajo durante el período de confinamiento del año 2020 motivado por la Covid-19 y practicar también la participación ciudadana en espacios virtuales a edades tempranas, las sesiones del 16 de abril de 2020 y del 25 de junio de 2020 se realizaron telemáticamente mediante la herramienta Zoom.

Desde su creación y hasta noviembre de 2021, este Grupo de Trabajo ha realizado un total de 19 sesiones. 
Figura 5. Dinámicas Grupo Trabajo Infantil CLIA

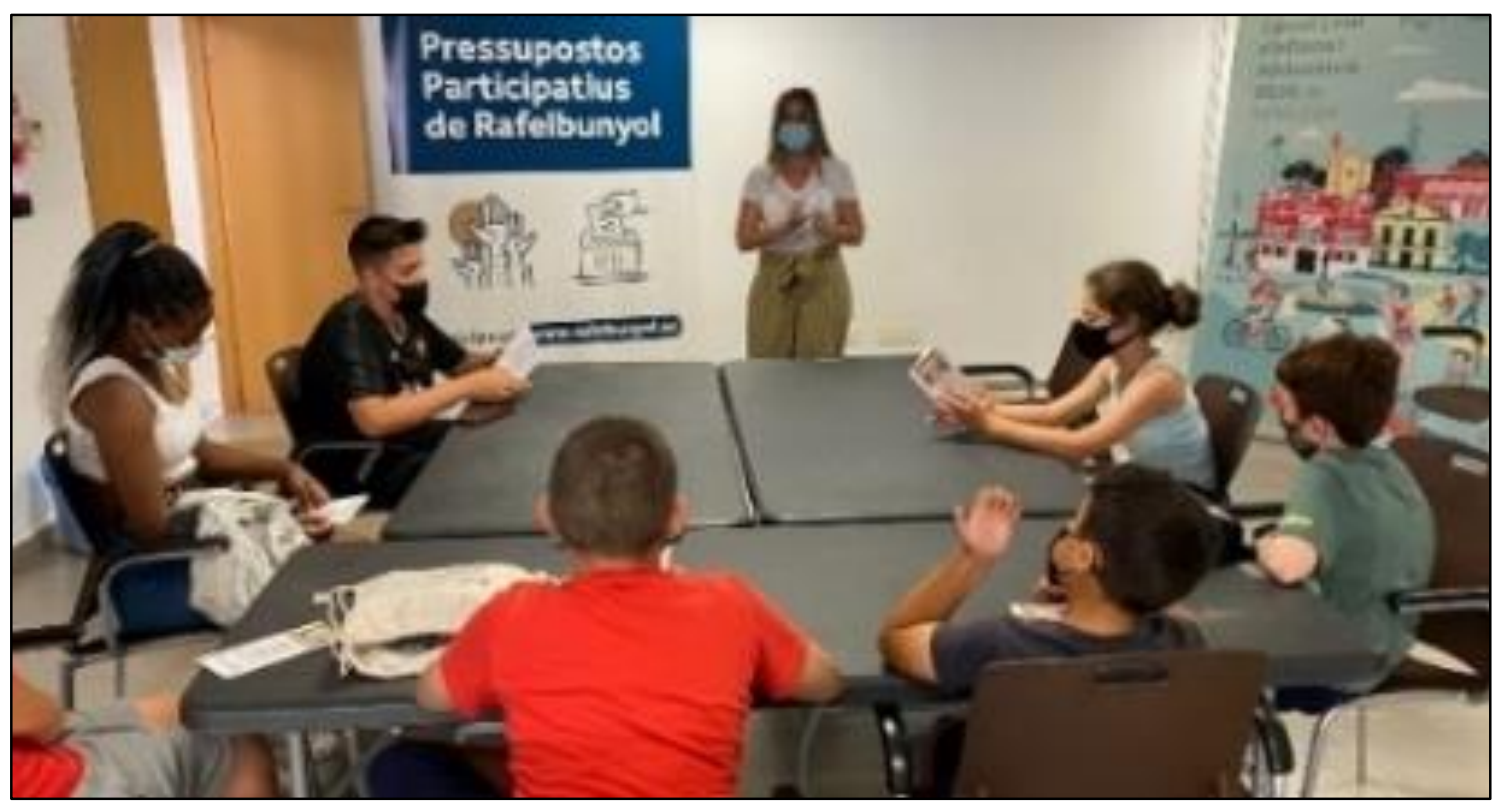

Fuente: elaboración propia.

\subsection{Foro de Participación Infantil y Juvenil}

De la misma manera, se realizó un Foro de Participación, como otro espacio de elaboración de propuestas y de intercambio de ideas, abierto a toda la infancia y adolescencia del municipio.

Durante la realización del mismo, las persones asistentes trasladaron sus opiniones, sugerencias y propuestas en cuanto a la gestión municipal y se reflexionó colectivamente sobre diferentes asuntos de interés público que interesan y/o preocupan a este colectivo.

Figura 6. Dinámicas Foro de Participación Infantil y Juvenil

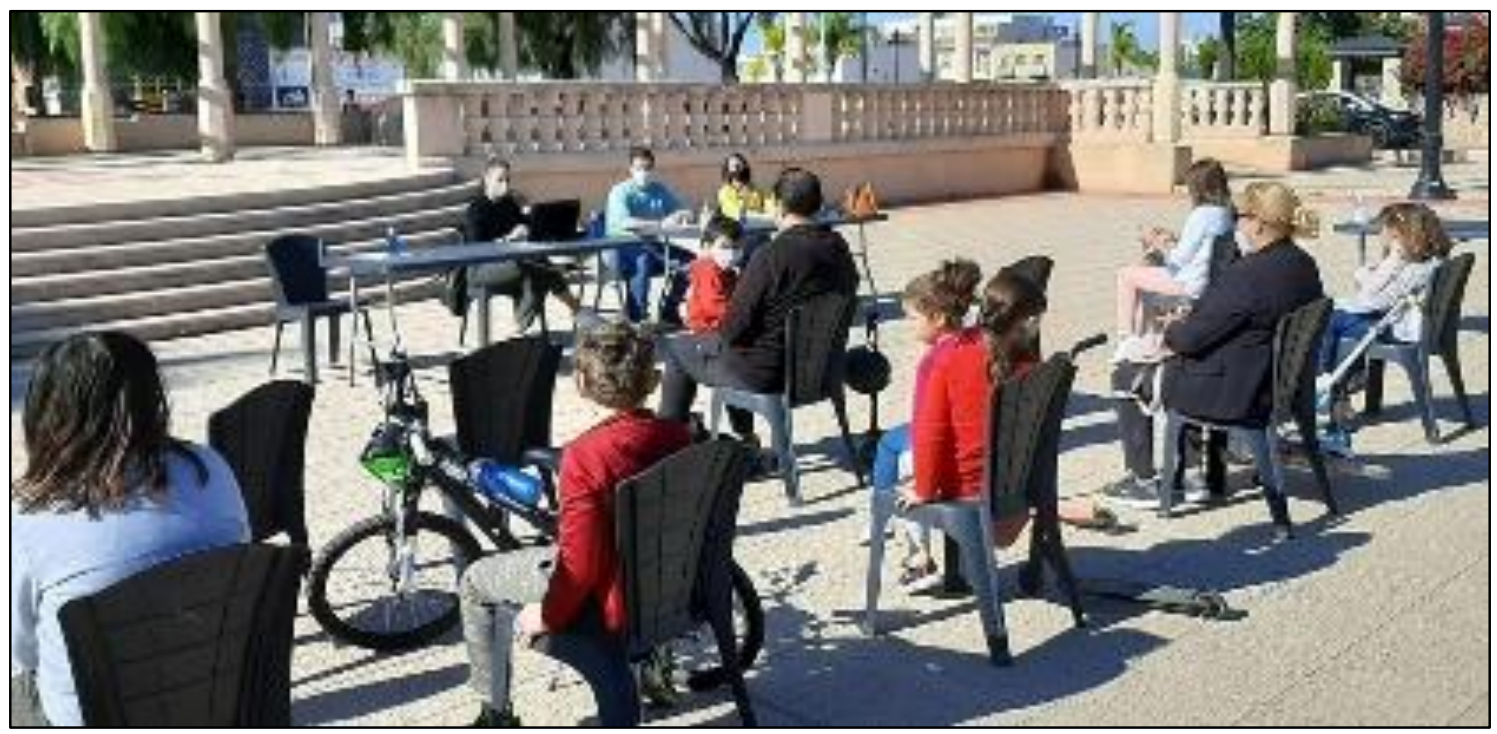

Fuente: elaboración propia.

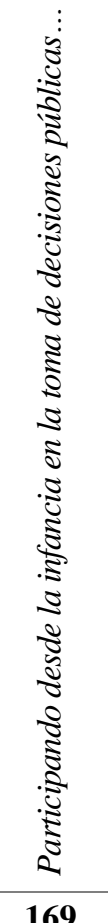


En este Foro participaron un total de 17 personas, de las cuales el 53\% fueron mujeres y el $47 \%$ hombres, y se recabaron 25 aportaciones ciudadanas, de las cuales el $72 \%$ fue realizadas por mujeres y el $28 \%$ por hombres.

\subsection{Elección infantil del graffiti de la Guardería Municipal}

Con la finalidad de continuar inculcando la práctica de hábitos democráticos relativos a la participación entre el colectivo infantil y de mitigar la tradicional exclusión de la infancia en la toma de decisiones públicas, el Ayuntamiento de Rafelbunyol delegó en los niños y niñas la elección de la temática del graffiti a realizar en la pared de la Guardería Municipal.

Para ello, en primer lugar, mediante la metodología participativa de dinámica de grupo, el Grupo de Trabajo Infantil del CLIA definió cinco opciones de temáticas (Racismo, Violencia de género, Lectura, Trabajo en equipo y Bullying) para este graffiti, que posteriormente fueron sometidas a un proceso de votación infanto-juvenil para su elección definitiva.

Esta votación, dirigida a las personas menores de 18 años de Rafelbunyol, se canalizó a través del Portal de Participación Ciudadana de Rafelbunyol (https://rafelbunyol.governalia.es/consultes/), lo que permitió, a su vez, que la infancia y adolescencia practicara la participación ciudadana en espacios virtuales.

Figura 7. Folleto informativo para la elección del graffiti de la Guardería Municipal

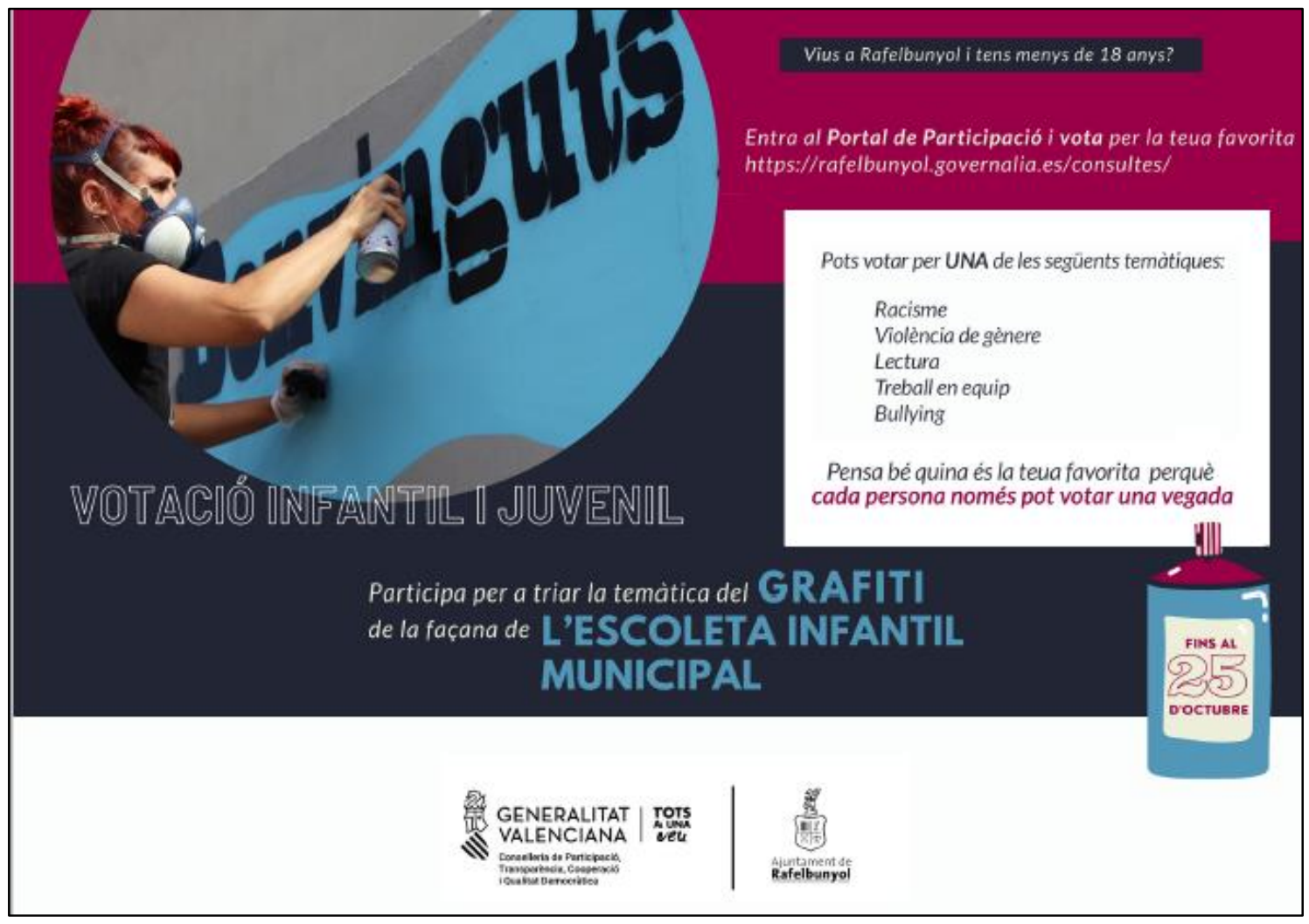

Fuente: elaboración propia. 
Asimismo, con la finalidad de fomentar la participación de la población infantil en este proceso, se realizaron folletos informativos que fueron difundidos de manera física en el Foro de Participación Infantil y en los centros educativos del municipio, y de manera telemática a través de las redes sociales y medios de comunicación del Ayuntamiento de Rafelbunyol.

En esta votación participaron un total de 47 personas (el 2,5\% del total de la población menor de 18 años $^{4}$ ), de las cuales el 53,2\% fueron niñas y el 46,8\% niños. Asimismo, de esas 47 personas participantes, el 48,78\% tenía entre 3 y 6 años, el 34,15\% entre 7 y 12 años y el 17,07\% entre 13 y 16 años.

\subsection{Presentación pública infantil sobre herramientas de participación}

Por último, con la finalidad de visibilizar e incrementar el protagonismo del colectivo infantil en la gestión municipal, tuvo lugar una presentación pública a cargo de los niños y niñas miembros del CLIA dirigida a la ciudadanía en general y a la población infantil y juvenil de Rafelbunyol en particular, en la que se dieron a conocer los órganos, espacios, procesos y herramientas existentes en el municipio para la participación ciudadana de este colectivo.

Figura 8. Presentación pública infantil sobre herramientas de participación

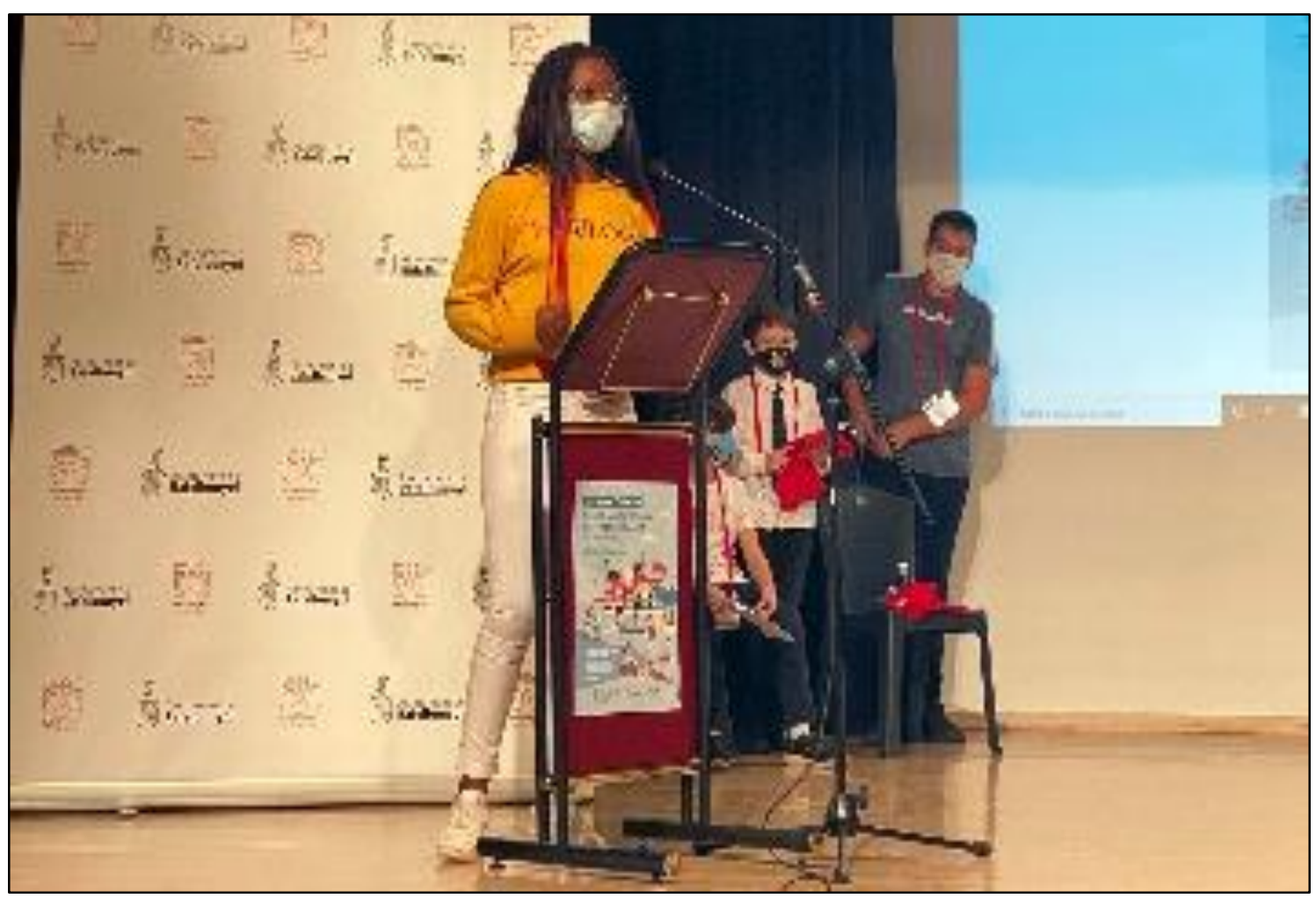

Fuente: elaboración propia.

\footnotetext{
${ }^{4}$ Un total de 1.879 personas empadronadas menores de 18 años según los datos del Padrón de 2019.
} 
Además, para mejorar la comprensión de estos aspectos por parte de la población infantil, se difundieron materiales informativos y divulgativos relacionados con la participación ciudadana en el municipio de este sector poblacional, elaborados con un lenguaje y diseño adaptado al colectivo infantil, como una versión adaptada del Reglamento del Consejo Local de Infancia y Adolescencia de Rafelbunyol, del Plan Municipal de Infancia y Adolescencia y un folleto informativo del CLIA.

Figura 9. Materiales divulgativos adaptados al colectivo infantil

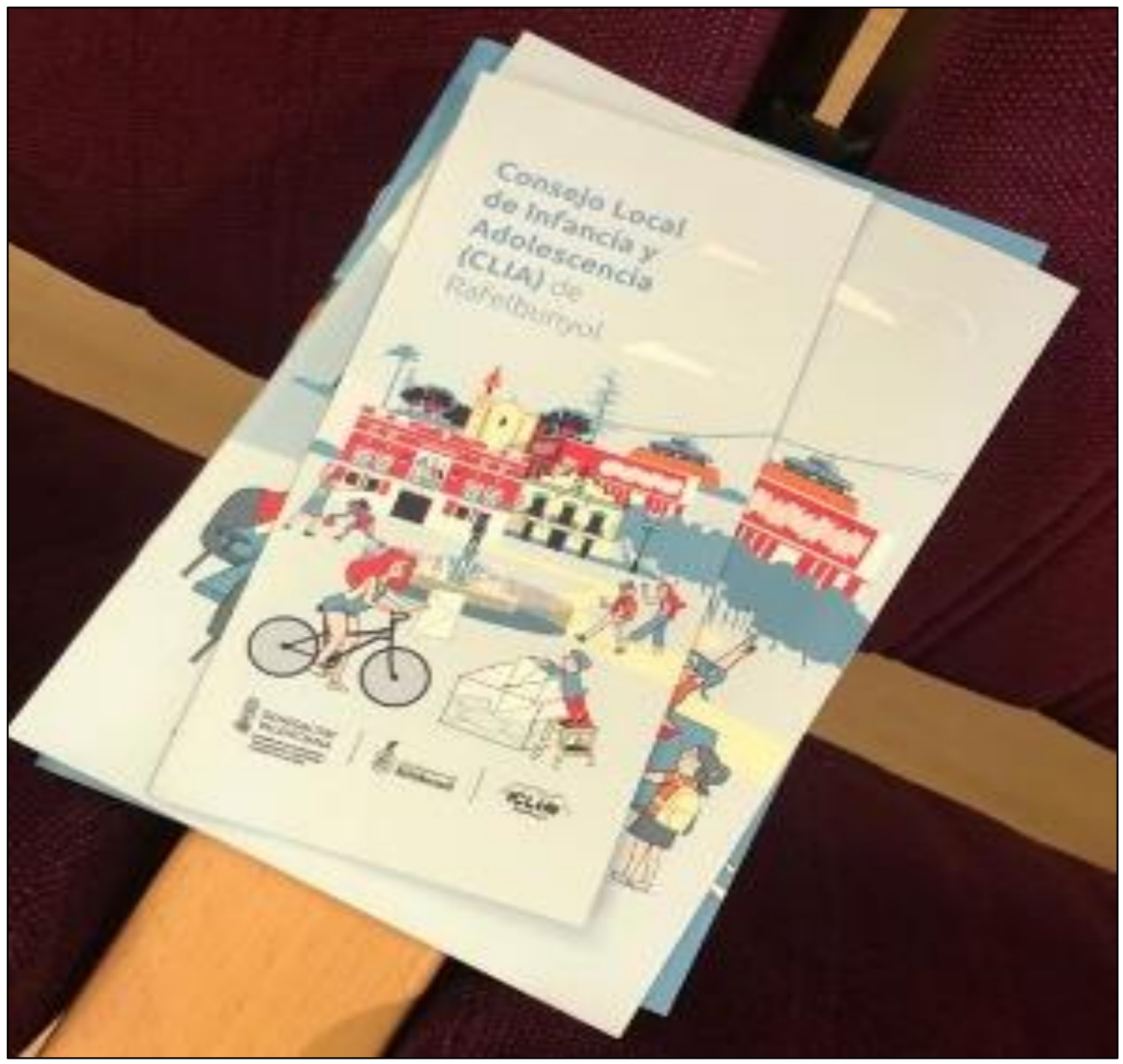

Fuente: elaboración propia.

En cuanto a los resultados de la presente estrategia en relación a los objetivos de la misma, destacar que la creación del Consejo Local de Infancia y Adolescencia (CLIA) de Rafelbunyol y del Grupo de Trabajo Infantil del CLIA (Actividades 2 y 3 ) se erigen como unos mecanismos que han permitido garantizar, de una manera prolongada en el tiempo, la participación ciudadana de la infancia y la adolescencia en la gestión pública, mitigando, por tanto, la tradicional exclusión por razón de edad de la población infantil en la toma de decisiones públicas. 
Este último aspecto, además, se vio reforzado mediante la elección por parte del alumnado del nombre oficial de su colegio (Actividad 1) y la elección infantil del graffiti de la Guardería Municipal (Actividad 5), en tanto que supusieron la participación directa y vinculante de este colectivo en la toma de unas decisiones públicas, delegando en ellos un poder real de decisión en cuanto a la gestión municipal.

Además, en su conjunto, las actuaciones realizadas durante la implementación de la presente estrategia han permitido inculcar y practicar entre el colectivo infantil hábitos democráticos relativos a la participación, fomentar la implicación ciudadana activa en las decisiones públicas desde edades tempranas y, especialmente, generar una cultura de gobernanza participativa y fortalecer el empoderamiento ciudadano desde la infancia.

\section{CONCLUSIONES}

Como se apunta en la introducción, la infancia es una etapa fundamental en la socialización y en el aprendizaje social, por lo que la implementación de una experiencia como la que aquí se expone, que ha permitido a este colectivo no solo la práctica de hábitos democráticos, sino también, participar de una manera activa, directa y vinculante en la gestión municipal, indiscutiblemente contribuye a su desarrollo como ciudadanos y, por tanto, a construir una ciudadanía empoderada y a generar una cultura de gobernanza participativa desde la infancia.

De hecho, en relación a estos dos último aspectos, la realización de las actuaciones de la presente estrategia como mecanismos de pedagogía e iniciación en el ejercicio de hábitos democráticos a edades tempranas, así como el impacto sociocultural de las mismas entre el colectivo infantil y adolescente del municipio, constituyen dos de los elementos más destacables de esta experiencia, junto con la práctica por parte de niños y niñas de la participación ciudadana en espacios virtuales a través de las Actividades 3 y 5.

Otro de los aspectos más destacables de la experiencia presentada es que ha permitido mitigar la tradicional exclusión por razón de edad de la población infantil en la participación de la toma de decisiones públicas, haciendo efectivo su derecho a participar y delegando en ellos un poder real de decisión, directo y vinculante.

En este sentido, apuntar también que la práctica entre el colectivo infantil de hábitos democráticos y de sus derechos, entre los que se encuentra el derecho a participar, implica una transformación de la tradicional representación adultocentrista que se tiene de la infancia como pre-ciudadanos, a su consideración como ciudadanos activos, que ejercen sus derechos y que contribuyen al bien común.

\section{REFERENCIAS}

Mayor, J. M., Molina, J., y Gómez, J. A. (2019). Promover el ejercicio del derecho a través de la educación: el Programa IRIS sobre Transparencia y los Presupuestos Participativos. Revista Española de la Transparencia, (9), 97-114. DOI: https://doi.org/10.51915/ret.49

Novella, A. M. (2012). La participación infantil: concepto dimensional en pro de la autonomía ciudadana. Revista Teoría de la Educación: Educación y Cultura en la 
Sociedad de la Información, 13(2), 380-403. DOI: https://doi.org/10.14201/eks.9015

Puig, J. M. (11 de diciembre de 2005). Lo necesitamos todo. El País. Recuperado de: https://elpais.com/diario/2005/12/11/opinion/1134255611_850215.html (20/11/2021).

UNICEF (2015). Convención sobre los Derechos del Niño. Recuperado de: https://ciudadesamigas.org/wp-content/uploads/2015/07/cdn_version20151.pdf $(18 / 11 / 2021)$. 